\title{
Consenting for Current Genetic Research: Views of Canadian Institutional Review Board Members
}

\section{Iris Jaitovich Groisman and Beatrice Godard}

Université de Montréal, Department of Social and Preventive Medicine, Canada

"Corresponding author: Beatrice Godard, Université de Montréal, Department of Social and Preventive Medicine, C.P. 6128, succ. Centre-ville, Montreal (Quebec), H3C 3J7, Canada, Tel: 15143436111 (\#44036); Fax: 1514343 5645; E-mail: Beatrice.godard@umontreal.ca

Received date: May 27, 2015; Accepted date: August 03, 2015; Published date: August 08, 2015

Copyright: $\odot 2015$ Groisman IJ. This is an open-access article distributed under the terms of the Creative Commons Attribution License, which permits unrestricted use, distribution, and reproduction in any medium, provided the original author and source are credited.

\begin{abstract}
The widespread use of next generation sequencing (NGS) technologies in genetic research protocols creates a need for sequencing material that has been already collected, be it for similar or different purposes, in addition to increasing the need for sharing both material and the genetic generated data to enrich study results. Along with this comes the need to recontact participants for a variety of reasons where consent for unanticipated use and obtaining additional health information are only some examples. Following an earlier content analysis on a sample of Canadian Institutional Review Boards (IRB) consent form templates and accompanying guidelines, and IRBapproved consent forms, we developed an online survey addressing the opinions and experiences of Canadian IRB members on the use of NGS in research. We report herein the results pertaining to secondary use, sharing of material and data and recontacting participants. Participants in our survey agree with the need for clear identification on the matter of secondary use on consent form documents as well as the importance of differentiating use of material from the generated data. Our responders seem to be unsure how these separate options could affect the research enterprise. Respondents to our study agree with study participants opinions obtained elsewhere about the importance of providing options when it comes to share their data. While all responders agree on the importance of increasing grounds for recontacting, not all accept a clause allowing researchers to contact participants by default and giving always the possibility to refuse any further communication at time of recontact. Furthermore, our survey allowed us to make connections between the information stated on consent documents resulting from our earlier analysis with member's views of said Boards. Adapting to new modalities of research is a challenging endeavour for IRB members. Continuous empirical research will allow the ethical oversight of research projects to keep pace with technological changes, simultaneously ensuring protection of human participants.
\end{abstract}

Keywords: Next generation sequencing; Informed consent; Genetic research; Ethical issues; Participants' protection; Institutional review boards; Secondary use; Data sharing; Recontacting participants.

\section{Background}

The prevalent use of next generation sequencing (NGS) technologies in genetic research protocols is changing the consent process necessary for participation in such projects [1,2]. As a consequence, there is a need to convey new information to research participants while preserving their autonomy in decision-making $[2,3]$. Earlier analysis on the use of NGS in research and the ethical and social issues surrounding participants' protection allowed us to identify several key themes impacting the consent process $[4,5]$. We identified the subjects of secondary or future use of material and data, sharing of material and data in relation to secondary use, and recontacting participants, among others $[2,4,5]$. These three topics are interrelated because they all require Institutional Review Boards (IRBs) and researchers to anticipate them, while they could be viewed as cloudy concepts by participants. A clear description on the part of researchers would allow participants' unambiguous decisions as to their involvement in a research project and how DNA - and the information derived from it - should be managed [2]. Furthermore, it would avoid any harm due to possible mishandling while capitalizing on its use [2].
Previous own work consisted in a content analysis using a sample of Canadian consent form templates and guidelines, and IRB-approved consent documents on these three topics and led us to propose the addition of well-defined categories for future use with a clear distinction between secondary use of material and the use of genetic data, the addition of options for sharing genetic data, and the widening of the grounds for recontacting research participants. The policy "Ethical Conduct for Research Involving Humans", known as the TriCouncil Policy Statement 2nd Edition (hereafter TCPS2), was used to frame our earlier analysis because it is applied by the three Canadian research agencies [6]. Investigators in Canada funded by any one of these research agencies are expected to comply with TCPS2 [2].

TCPS2 grants a pivotal role to IRBs and its members in the management of secondary use of biological material and/or data, the way in which data is shared, and the contact between investigators and research participants [6]. Although revisions to the TCPS undergo open consultations, we deemed it important to examine the views and experiences of Canadian IRB members on a series of subjects pertaining to the use of NGS in research: we developed and used an online survey based on the outcomes of our earlier research and on related studies that were conducted elsewhere with IRB members and researchers [2,4,5,7-9]. We report herein the views of Canadian IRB members' participants of our study on the matters of secondary use, sharing of material and data and recontacting participants. IRB members' opinions on the returning of genetic results - both related 
Page 2 of 4

and unrelated to the matter that prompts a research study - are being reported elsewhere.

\section{Methods}

\section{Sample and recruitment}

We invited Canadian IRB members to participate in an anonymous web-based survey by sending a letter of invitation - containing a link to said survey - to IRB coordinators and IRB chairs whose contact information was publicly available, asking to distribute the invitation among their Boards' voting members. The Canadian Association of Research Ethics Boards (CAREB) and the Canadian Association of Research Administrators (CARA) distributed via email the invitation among their associates. In all scenarios, reminder emails were sent twice about two weeks apart.

An invitation to participate was also posted on these associations' LinkedIn sites, as well as on the Quebec Ministry of Health, Ethics and Quality Directorate website. The invitation letter and survey were available in English and in French. At the end of the survey respondents were requested to forward the invitation to other IRB members in an attempt to increase the number of participants.

\section{Survey development and data collection}

To inform survey development on the subjects presented in this work, we used results from previous own research on the use of NGS in research on mental health and brain disorders $[2,5,10]$. The survey contained multiple choice questions distributed in six areas in addition to demographic information of participants. We report herein our results related to secondary use and sharing of material and data and recontacting participants. Questionnaire on-lining, data collection and statistical analysis were conducted by the IT department at University of Montreal Public Health Research Institute. Data collection was anonymous to the researchers conducting this study. Geographical location and role of board member and/or board characteristics were not combined to decrease possibility of identification. This study and all the accompanying documents were approved by the Health Science Research Ethics Board from University of Montreal. The questions pertaining to the data presented herein are available upon request.

\section{Statistical analysis}

Descriptive statistics produced a portrait of IRB members' opinions and views on the matters of secondary use and sharing of material and data and recontacting participants. Data were analyzed using SPSS version 22 (IBM, Somers, NY). Finally, sample sizes varied by question because participants were allowed to skip any question they did not wish to answer.

\section{Results}

Participants in our survey hold different positions on their boards, which belong - in their majority - to hospitals, and universities and academic centers (Table 1).

Respondents were given the possibility of answering where in Canada they are located. Only participants from Quebec, Ontario, Nova Scotia, Saskatchewan and British Columbia, out of the 10 provinces and 3 Canadian territories identified their geographical location. A majority $(66.7 \%$ of 81$)$ declared having experience conducting research with human subjects and among those, some (27.5\%) also had experience conducting genetic/genomic research.

\begin{tabular}{|l|l|l|}
\hline Position on a REB (n=76) & $\mathbf{n}$ & Percentage \\
\hline Chair & 16 & 21.1 \\
\hline Jurist/ Ethicist & 11 & 14.5 \\
\hline Member of the community & 14 & 18.4 \\
\hline Scientific member & 20 & 26.3 \\
\hline Other & 15 & 19.7 \\
\hline Work setting & & \\
\hline Hospital & 39 & 31.0 \\
\hline Academic Medical Centre & 25 & 19.8 \\
\hline University & 36 & 27.8 \\
\hline Government & 12 & 9.5 \\
\hline Private & 9 & 6.3 \\
\hline Other & 7 & 5.6 \\
\hline
\end{tabular}

Table 1: Participants' characteristics.

A total of 52 IRB members responded that the subject of secondary use is explained on their board's documents such as consent form templates, IRB guidelines or both. Secondary use is clearly indicated with a title or subtitle in $76.6 \%$ of the respondents' IRB documents (76.6\% among the 47 that answered this question).

Eighty five percent (85.2\% of 54 respondents) preferred that the subject of secondary use appear in a separate section with its own title or subtitle on the consent form. When participants were asked if the explanation of secondary use of material is presented separately from the explanation of secondary use of the generated genetic data on their board's documents, their answers show a close distribution between "yes" (28.3\%), "no" (37.7\%) and "I don't know"(24.5\%) among the 53 respondents to this question (Figure 1).

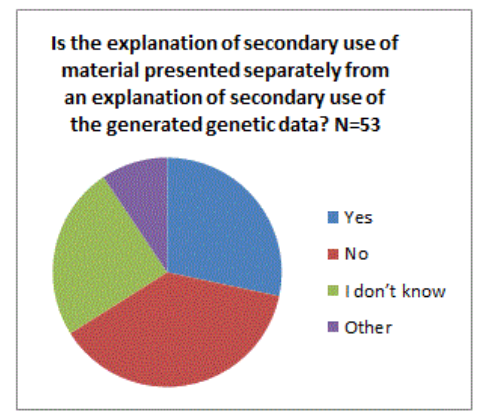

Figure 1: Secondary use

IRB members considered that explanations about the difference between secondary use of genetic material and secondary use of genetic data are important for research participants' informed consent $(88.5 \%, \mathrm{~N}=51)$. When asked if giving the explanation (and hence the 
option) of secondary use of material separately from secondary use of the generated genetic data would limit research projects, respondents $(\mathrm{N}=51)$ showed divided opinions between yes (37\%), no (33\%) and not knowing (25\%) what the outcome would be (Table 2).

\begin{tabular}{|c|c|c|c|c|c|c|c|c|}
\hline \multirow{2}{*}{$\begin{array}{l}\text { Do you consider } \\
\text { explanations to } \\
\text { research } \\
\text { participants about } \\
\text { the difference } \\
\text { between } \\
\text { secondary use of } \\
\text { genetic material } \\
\text { and secondary use } \\
\text { of genetic data to } \\
\text { be }\end{array}$} & \multicolumn{2}{|c|}{ Yes } & \multicolumn{2}{|c|}{ No } & \multicolumn{2}{|c|}{ I don't know } & \multicolumn{2}{|c|}{ Other } \\
\hline & $\%$ & $\mathrm{~N}$ & $\%$ & $\mathrm{~N}$ & $\%$ & $N$ & $\%$ & $\mathrm{~N}$ \\
\hline $\begin{array}{l}\text { Important for } \\
\text { participants' } \\
\text { informed consent } \\
(\mathrm{N}=52)\end{array}$ & 88.5 & 46 & 5.8 & 3 & 3.8 & 2 & 1.9 & 1 \\
\hline $\begin{array}{l}\text { Limiting } r \\
\text { research projects } \\
(\mathrm{N}=41)\end{array}$ & 37.3 & 19 & 33.3 & 17 & 25.5 & 13 & 3.9 & 2 \\
\hline
\end{tabular}

Table 2: Secondary use of material and of data

According to our participants' responses, consent forms offer a diverse variety of choices when it comes to secondary use of material and data such as exclusive secondary use of material, or of data, secondary use of both as one entity, or secondary use of both but in a completely separated way.

We asked IRB members if they considered appropriate that research participants be given the possibility to choose if they want that their genetic data be shared, either in public or in restricted databases. Almost $89.7 \%(\mathrm{~N}=78)$ of respondents agreed that it was correct to offer this choice, although there was no consensus on whether this option would limit the research enterprise (Table 3).

\begin{tabular}{|l|l|}
\hline $\begin{array}{l}\text { Is it appropriate to provide participants with options } \\
\text { on genetic data sharing? } \\
\text { (i.e. public or restricted data bases) }(\mathbf{N}=\mathbf{7 8})\end{array}$ & Percentage \\
\hline Yes & 89.7 \\
\hline No & 10.3 \\
\hline $\begin{array}{l}\text { Do you consider this option to limit research enterprises? } \\
\text { (N=68) }\end{array}$ & \\
\hline Yes & 30.9 \\
\hline No & 54.4 \\
\hline Other & 14.7 \\
\hline
\end{tabular}

Table 3: Data sharing

The majority also considered unlikely that coded genetic data could help an unauthorized third party personally identify $(67.6 \% \mathrm{~N}=74)$ or harm $(64 \% \mathrm{~N}=75)$ an individual.

In genetic/genomic research, there are a number of reasons that show it is important to re-contact study participants at any point in a project's development: secondary use of samples and/or data, obtaining further health information, obtaining more samples, returning individual research results (namely those related to the matter prompting the study), providing information on incidental findings, and recruitment. All of these subjects were equally important to respondents. However, when asked if researchers should be able to re-contact participants in all cases provided that participants would be free to refuse to follow up on this re-contact, almost $40 \%$ of respondents still answered "No" (Figure 2).

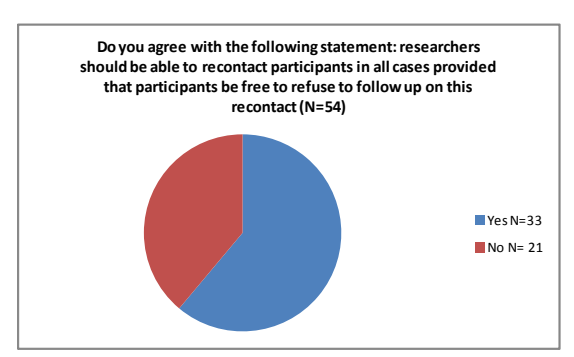

Figure 2: Recontacting participants

\section{Discussion}

IRB members participating in our survey who responded to questions on the matters of secondary use indicated that on their IRB documents, the subject of secondary use is clearly indicated. This result differs from our earlier findings on IRB consent form templates and associated guidelines, and IRB approved documents. In our earlier study, the subject of secondary use was not broached in a systematic way from one unit of analysis to another [2]. While our sample size in the present study is small, it is also possible that those who answered this question are coincidentally on the same boards whose documents clearly addressed secondary use. In addition, the period that elapsed between the two studies was sufficient for an IRB to have updated their guidelines and templates with language and message accommodating the needs of current genetic research. Participants' protection is paramount to IRB members, so presenting the matter of secondary use in a clear systematic way will eventually facilitate understanding on the part of participants and hence autonomous decisions. However, it is not clear to the participants of our survey how these explanations and thus the options for secondary use - would impact the availability of data necessary for collaborative genetic studies. Results from a survey among IRB administrators conducted by Goldberg et al explain that when it comes to secondary use in genetic research, IRBs are not consistent in their "protocol review, risk assessment, and data sharing, especially when specimens from biobanks are not anonymized" [11]. The majority of respondents to questions on the matter of data sharing are in favour of providing options for this endeavour. IRB members' position thus coincides with reports on participants' views and preferences on deciding how their personal information will be shared, and who would have access to it [12]. In our earlier analysis we noticed that in IRB-approved documents, data sharing was part of the study and compulsory [2]. When compulsory, the option for participants is to decide whether or not to take part in the study. By making participation conditional on the willingness to consent to data sharing, researchers may be losing individuals that do not want their genetic information to be used by other researchers or other institutions or even used in countries other than the ones who are conducting the initial project. Guaranteeing individuals' privacy and confidentiality and providing a proper and clear explanation of the societal benefits of 
Page 4 of 4

data sharing are then crucial matters in having research participants consenting to share their information. Miller et al collected information on the views of European scientists that are involved in multi-national European genetic projects about having their own genome sequenced. This population - who undoubtedly understands the subject matter - expressed very diverse positions towards data sharing that vary from "highly protective" to open sharing through databases. However, they agreed on the importance of clear information to participants in genetic research as well as providing explanations on the variety of uses of genetic data [13].

While researchers may need to communicate with participants for a wide variety of reasons, having the possibility of recontacting for participation in future research is a recruitment strategy that allows researchers to get in touch with potential participants who have already shown interest to partake in a research enterprise. It is expected that researchers inform prospective participants why they are being contacted, "how information about them was obtained, and what will happen to that information if they decide not to participate." [14]. Our previous study on IRB documentation showed that the main reason for recontacting participants was to obtain consent for secondary use. In a minority of cases it was to return results or to update health information. IRB members, however, consider almost equally important a wide variety of reasons for being in touch with research participants, more in line with the current needs of genetic research. Based on our position, which is in accordance with Beskow et al., we asked if IRB members agreed with advising participants that "recontacting will occur", thereby reminding them that the option to refuse to be informed of the reason that prompts any recontact should always be open $[2,15]$. Although respondents to our survey give similar importance to all the reasons that were presented to them for recontacting participants, not all agree with our position. This highlights the significance of how the message about being recontacted is conveyed to participants, and shows the critical role that the communication between investigators and research participants plays in current genetic research.

\section{Conclusion}

Our results pertain to the views of Canadian IRB members that confront challenges characteristic to the use of NGS in research. Because of the limited number of responders to our survey we can't conclude that the views presented in this work are representative of Canadian IRB members as a whole. However, adapting to new modalities of research is a challenging endeavour for IRB members everywhere. We were able to link how information on secondary use, sharing of material and data, and recontacting participants is conveyed in earlier analysis on a sample of Canadian IRB documents, with the opinion of a group of Canadian IRB members and with results published elsewhere on the same subjects among the public and scientists [11-13]. The latter confirms the relevance on the topics and information independently of type and number of participants, and their geographical location.

Our results taken together with others confirm the need of clear information to participants and a more systematic approach to research review on the part of IRBs. It is clear then that there is a need for continuous empirical research in order to allow the ethical oversight of research projects to keep pace with technological changes, simultaneously ensuring protection of human participants.

\section{Competing interests}

BG declares no competing interests. IJG serves as a member and consultant on ethics committees in the public and private sectors.

\section{Acknowledgements}

We thank Mr. Thierry Hurlimann and Ms. Nathalie Egalité for helpful discussion, and Nathalie for revising the manuscript. We are indebted to the Canadian Association of Research Ethics Boards, the Canadian Association of Research Administrators, and the Quebec Ministry of Health, Ethics and Quality Directorate for their help in distributing the survey, and to all IRB members responding to our survey.

\section{References}

1. Caulfield T, McGuire AL, Cho M, Buchanan JA, Burgess MM, et al. (2008) Research ethics recommendations for whole-genome research: consensus statement. PLoS biology 6: e73.

2. Jaitovich Groisman I, Egalite N, Godard B (2014) Consenting for current genetic research: is Canadian practice adequate? BMC medical ethics 15: 80.

3. Tabor HK, Stock J, Brazg T, McMillin MJ, Dent KM (2012) Informed consent for whole genome sequencing: a qualitative analysis of participant expectations and perceptions of risks, benefits, and harms. Am J Med Genet 158A: 1310-1319.

4. Groisman IJ, Mathieu G, Godard B (2012) Use of next generation sequencing technologies in research and beyond: are participants with mental health disorders fully protected? BMC medical ethics 13: 36 .

5. Mathieu G, Groisman IJ, Godard B (2013) Next generation sequencing in psychiatric research: what study participants need to know about research findings. Int J Neuropsychopharmacol 16: 2119-2127.

6. Research Council of Canada, Tri-Council Policy Statement: Tri-Council Policy Statement: Ethical Conduct for Research Involving Humans (TCPS 2). (2010) Canadian Institutes of Health Research NSaERCoC, and Social Sciences and Humanities

7. Dressler LG, Smolek S, Ponsaran R, Markey JM, Starks H et al. (2012) IRB perspectives on the return of individual results from genomic research. Genet Med 14: 215-222.

8. Keane MA (2008) Institutional review board approaches to the incidental findings problem. J Law Med Ethics 36: 352-355, 213.

9. Simon CM, Williams JK, Shinkunas L, Brandt D, Daack-Hirsch S et al. (2011) Informed consent and genomic incidental findings: IRB chair perspectives. J Empir Res Hum Res Ethics6: 53-67.

10. Egalite N, Groisman IJ, Godard B (2014) Genetic counseling practice in next generation sequencing research: implications for the ethical oversight of the informed consent process. J Genet Couns 23: 661-670.

11. Goldenberg AJ, Maschke KJ, Joffe S, Botkin JR, Rothwell E et al. (205) IRB practices and policies regarding the secondary research use of biospecimens. BMC medical ethics 2015, 16: 32.

12. Ludman EJ, Fullerton SM, Spangler L, Trinidad SB, Fujii MM et al. (2010) Glad you asked: participants' opinions of re-consent for dbGap data submission. J Empir Res Hum Res Ethics 5: 9-16.

13. Miller J, Soulier A, Bertier G, Cambon-Thomsen A (2014) Professionals' attitudes regarding large-scale genetic information generated through next generation sequencing in research: a pilot study. Journal of empirical research on human research 9: 56-58.

14. Beskow LM, Botkin JR, Daly M, Juengst ET, Lehmann LS, et al. (2004) Ethical issues in identifying and recruiting participants for familial genetic research. American journal of medical genetics 130A: 424-431.

15. Beskow LM, Namey EE, Cadigan RJ, Brazg T, Crouch J (2011) Research participants' perspectives on genotype-driven research recruitment. J Empir Res Hum Res Ethics 6: 3-20. 